\title{
Occlusion of uncommon anatomic variant of thalamic blood supply (so-called artery of Percheron) presenting as bilateral thalamic infarcts
}

\author{
Authors: Mahmood Bayan, Suleman Kanani, Alexandra Lupu, Jelena Sergentani and Daniel Varga
}

\section{Background}

Two cases of bilateral thalamic infarction are discussed. Both patients presented similarly with decreased consciousness and visual disturbance within a 5-month period of a stroke rotation.

\section{Summary}

Although infarcts restricted to the thalamus were reported for the first time more than 100 years ago by Dejerine and Roussy, they remain an uncommon presentation of stroke and account for only $11 \%$ of all vertebrobasilar infarcts. Bilateral thalamic infarction is rare, accounting for only $0.6 \%$ of all cerebral infarctions, and results from a combination of predisposing factors and anatomic variations. The pattern of bilateral thalamic stroke is usually seen after paramedian artery infarct of the so-called artery of Percheron (AOP); this is a rare anatomical variant of thalamic blood supply arising from the posterior cerebral artery, providing bilateral arterial supply to the paramedian thalami and the rostral midbrain. AOP occlusion accounts for $4-18 \%$ of all thalamic strokes and $0.1-2 \%$ of all ischaemic strokes.

The thalamus is involved in several functions of the body including regulation of sleep and wakefulness, motor control, receiving auditory, somatosensory and visual sensory signals, and relaying sensory signals to the cerebral cortex. Thalamocortico-thalamic circuits are involved in consciousness, arousal, level of awareness, and activity. Patients may take days to weeks to recover from a thalamic infarct and seem to be in a sleep-like state. In these cases, both patients were noted to be sleeping for the entire day and, as somnolence cleared, vertical gaze palsy became apparent.

\section{Conclusion}

Clinical correlation and appropriate imaging are essential to diagnose bilateral thalamic infarctions. Although it has been reported in literature that it is rare to see this type of infarction, a review of recent case reports and our experience based on these case presentations raise the question of whether bilateral thalamic infarcts are really rare or simply underdiagnosed. 